A. Y. S. Cheng - A. P. S. Kong • V. W. S. Wong

W. Y. So - H. L. Y. Chan - C. S. Ho - C. W. K. Lam •

J. S. Tam - C. C. Chow $\cdot$ C. S. Cockram •

J. C. N. Chan - P. C. Y. Tong

\title{
Chronic hepatitis B viral infection independently predicts renal outcome in type 2 diabetic patients
}

Received: 23 November 2005 / Accepted: 29 March 2006 / Published online: 31 May 2006

(C) Springer-Verlag 2006

\begin{abstract}
Aims/hypothesis: We examined the association between chronic hepatitis B virus (HBV) infection and clinical outcomes in a consecutive cohort of Chinese patients with type 2 diabetes. Subjects, materials and methods: Between 1995 and 1999, 2,838 type 2 diabetes patients underwent comprehensive assessments and blood screening for hepatitis B surface antigen (HBsAg). The risk of occurrence of cardiovascular events and end-stage renal disease (defined as need for dialysis, doubling of serum creatinine or serum creatinine $\geq 500 \mu \mathrm{mol} / \mathrm{l}$ ) was compared between HBsAg-positive and HBsAg-negative groups. Results: At baseline, HBV-infected patients $(n=286,10.1 \%)$ were younger ( $51.0 \pm 11.5$ vs $53.7 \pm 12.7$ years, $p=0.004)$, had earlier onset of diabetes $(51.0 \pm 11.5$ vs $53.7 \pm 12.7$ years, $p=0.001)$ and a higher frequency of retinopathy ( 28 vs $22 \%$, $p=0.03$ ) than non-HBV-infected patients. After a median follow-up of 3.5 years (interquartile range: $1.7-5.9$ years) and
\end{abstract}

A. Y. S. Cheng · A. P. S. Kong · V. W. S. Wong · W. Y. So ·

H. L. Y. Chan - C. C. Chow - C. S. Cockram -

J. C. N. Chan $(\bowtie) \cdot$ P. C. Y. Tong

Department of Medicine and Therapeutics,

The Chinese University of Hong Kong,

Prince of Wales Hospital,

Shatin, NT, Hong Kong SAR, People's Republic of China

e-mail: jchan@cuhk.edu.hk

Tel.: +852-2632-3138

Fax: $+852-2632-3108$

C. S. Ho · C. W. K. Lam

Department of Chemical Pathology,

The Chinese University of Hong Kong,

Prince of Wales Hospital,

Shatin, Hong Kong, People's Republic of China

J. S. Tam

Department of Microbiology,

The Chinese University of Hong Kong,

Prince of Wales Hospital,

Shatin, Hong Kong, People's Republic of China

A. P. S. Kong

Li Ka Shing Institute of Health Sciences,

The Chinese University of Hong Kong,

Prince of Wales Hospital,

Shatin, Hong Kong, People's Republic of China adjustment of age, glycaemic control and other potential confounding factors, $\mathrm{HBV}$-infected patients were more likely to develop end-stage renal disease than non-HBV infected patients (8.7 vs $6.4 \%)$ with a hazard ratio of $4.5(95 \% \mathrm{CI}$ 1.1-18.6). The difference in the frequency of cardiovascular endpoints was not statistically significant. Conclusions: In Chinese type 2 diabetes patients, chronic HBV infection was associated with increased risk of end-stage renal disease, and this was independent of other potential confounding factors. Early identification of HBV status and close surveillance of renal function are important in patients with type 2 diabetes who are living in areas where HBV is endemic or who are at risk of chronic $\mathrm{HBV}$ infection.

Keywords Chinese $\cdot$ Hepatitis B viral infection - Renal outcome - Type 2 diabetes

Abbreviations ACR: albumin:creatinine ratio - ESRD: end-stage renal disease - eGFR: estimated glomerular filtration rate $\mathrm{HBV}$ : hepatitis $\mathrm{B}$ virus $\cdot \mathrm{HCV}$ : hepatitis $\mathrm{C}$ virus $\cdot \mathrm{HBsAg}$ : hepatitis $\mathrm{B}$ virus surface antigen $\cdot \mathrm{HBeAg}$ : hepatitis B virus e-antigen - TC: total cholesterol - TG: total triglycerides

\section{Introduction}

Both type 2 diabetes mellitus and chronic hepatitis B virus (HBV) infection are major public health problems in Asia, including Hong Kong. In a population-based survey, conducted in Hong Kong between 1995 and 1996, the prevalence of type 2 diabetes was approximately $10 \%$ [1]. A similar proportion of our population also suffers from chronic HBV infection [2]. The liver is a key organ in intermediary metabolism and plays a pivotal role in the pathogenesis of insulin resistance. Hence, glucose intolerance is common in patients with liver cirrhosis of whatever aetiology, and around $20 \%$ of cirrhotic patients have overt diabetes [3]. Chronic hepatitis $\mathrm{C}$ virus (HCV) infection is well known to be associated with increased risk of type 2 diabetes [4-10]. This risk association is independent of the 
presence of cirrhosis [11, 12], and has been hypothesised to be mediated through insulin resistance and chronic inflammation [12]. HCV infection has been shown to be a predictor of worse renal survival in African-American diabetic patients [13] and is significantly associated with decline of renal function in Japanese patients with diabetes [14].

Compared with HCV infection, the associations between HBV infection, type 2 diabetes and its complications are less well documented. In a retrospective study of 767 pregnant Chinese women, chronic HBV infection was associated with a 3.5-fold increased risk of gestational diabetes [15]. Women with chronic HBV infection also had higher plasma glucose levels than those who were uninfected at their first antenatal visit. In a small cohort of Chinese patients with type 2 diabetes and overt nephropathy that was previously followed up by our group, HBV carrier status was associated with increased risk of cardiovascular events [16]. Based on these findings, we hypothesised that HBV infection might be associated with an increased risk of type 2 diabetes and its complications. In this prospective analysis, we examined the association between chronic HBV infection and its association with clinical outcomes in a cohort of Chinese patients with type 2 diabetes.

\section{Subjects, materials and methods}

\section{Subjects}

The Prince of Wales Hospital is the teaching hospital of the Chinese University of Hong Kong and serves a population of over 1.2 million. Since 1995, as part of a continuous quality improvement programme, all diabetic patients newly referred to the hospital and those under regular review at our medical clinics have undergone comprehensive assessments of complications and risk factors based on the European DiabCare protocol, both at first visit and at periodic intervals of 2-3 years [17].

When the programme was first introduced in 1995, in addition to newly referred patients, those who had been followed up at our clinic and who had no major cardiovascular or renal complications were among the first to undergo comprehensive assessments before being referred back to the community for shared care with their family doctors. In a consecutive cohort of 2,838 patients with type 2 diabetes who were recruited between 1995 and 1999, we examined the status of chronic HBV infection by detecting the presence of HBV surface antigen (HBsAg) in the stored serum. Informed consent was obtained from all patients and the study was approved by the Chinese University of Hong Kong Clinical Research Ethics Committee. Patients with type 1 diabetes, defined as those presenting with diabetic ketoacidosis, acute presentation with heavy ketonuria $(>3+)$ or continuous requirement of insulin within 1 year of diagnosis were excluded from the present analysis [18].

\section{Clinical and metabolic measurements}

Clinical assessments including documentation of demographic data, anthropometric measurements and diabetic complications were performed at baseline. Macrovascular complications included peripheral vascular disease, history of stroke, myocardial infarction, angina and heart failure requiring hospitalisation, and revascularisation procedures. Peripheral vascular disease was identified by the absence of foot pulses on palpation, confirmed by Doppler ultrasound examination of the ankle-to-brachial ratio of $<0.9$. Fundi were examined by a physician through a dilated pupil for the presence of the typical changes of diabetic retinopathy, including exudates, haemorrhages and laser scars. Peripheral sensory neuropathy was diagnosed if two of the following findings were present: reduced sensation to monofilament examination in any part of the sole with normal skin, a score $\leq 6 / 8$ (age $\leq 65$ years) or $\leq 4 / 8$ (age $>65$ years) using the graduated tuning fork, or typical symptoms of numbness or abnormal sensation over both lower limbs.

Fasting blood samples were taken for measurement of glucose, lipid profile (including total cholesterol [TC], HDL cholesterol, triglycerides [TG] and calculated LDL cholesterol), renal and liver functions and HBsAg. A sterile, random spot urine sample was used to measure albumin:creatinine ratio (ACR) followed by a timed collection (4-h or 24-h) for albumin excretion rate. Using the ACR from these two samples, normoalbuminuria was defined as a mean ACR $<3.5 \mathrm{mg} / \mathrm{mmol}$, microalbuminuria was defined as an ACR between 3.5 and $25 \mathrm{mg} / \mathrm{mmol}$, and macroalbuminuria was an ACR of $\geq 25 \mathrm{mg} / \mathrm{mmol}$ [19]. The estimated glomerular filtration rate (eGFR; expressed in $\mathrm{ml}$ $\min ^{-1} 1.73 \mathrm{~m}^{-2}$ ) was calculated using the modified Modification of Diet in Renal Disease equation [20].

In 2000, the clinical data were censored by the Hong Kong Death Registry and Hospital Authority hospital records using the Ninth International Classification of Diseases codes. All Hong Kong residents have a unique identity card number, which was used to track this information. Details of all admissions with primary and secondary diagnoses and last available plasma creatinine results were retrieved from the Central Computerised System at the Hospital Authority Head Office. All causes of death were ascertained by review of the case notes by an endocrinologist. Renal endpoint was defined as those requiring dialysis, doubling of baseline serum creatinine or serum creatinine level $\geq 500 \mu \mathrm{mol} / 1$.

\section{Laboratory assays}

Plasma glucose was measured by a hexokinase method using an automated analyser (Hitachi 911; Boehringer Mannheim, Mannheim, Germany). $\mathrm{HbA}_{1 \mathrm{c}}$ was measured using an automated ion-exchange chromatographic method (Bio-Rad Laboratory, Hercules, CA, USA; reference range $5.1-6.4 \%$ ). The inter- and intra-assay $\mathrm{CV}$ for $\mathrm{HbA}_{1 \mathrm{c}}$ was $\leq 3.1 \%$ at values $<6.5 \%$. Total cholesterol, TG and HDL- 
cholesterol were measured by enzymatic methods on the Hitachi 911 automated analyser using reagent kits supplied by the manufacturer. LDL-cholesterol was calculated by Friedewald's equation for TG $<4.5 \mathrm{mmol} / 1$ [21]. The precision performance of these assays was within the manufacturer's specifications. Urinary creatinine (Jaffe's kinetic method) and albumin (immunoturbidimetry method) were also measured on the Hitachi 911 analyser using reagent kits supplied by the manufacturer. The inter-assay precision CVs were 12.0 and $2.3 \%$ for urinary albumin concentrations of 8.0 and $68.8 \mathrm{mg} / \mathrm{l}$, respectively. The lowest detection limit was $3.0 \mathrm{mg} / \mathrm{l}$. Plasma creatinine (Jaffe's kinetic method) was measured on a Dimension AR system (Dade Behring, Deerfield, IL, USA). A complete blood profile, including white cell count, was measured using an automated cell counter (GEN-S; Beckman Coulter, Miami, FL, USA). HBsAg was tested using commercially available ELISAs (Abbott Diagnostika, Wiesbaden-Delkenheim, Germany).
Statistical analyses

Data were analysed using the Statistical Package for Social Sciences (SPSS version 11.5). Plasma TG, creatinine and albuminuria were logarithmically transformed because of skewed distributions. All data are expressed as mean \pm SD or median (interquartile range) as appropriate. Student's $t$ test or analysis of covariance (ANCOVA) was used for between-group comparisons for continuous variables, and the $\chi^{2}$ test was used for categorical variables. A Coxregression model was used to estimate the hazard ratio with 95\% CI for mortality and clinical endpoints, with the assumption that the effects of different variables on survival were constant. For the mortality and clinical endpoints, multivariate analysis was performed using age, sex, age at onset of diabetes, smoking, blood pressure, body mass index, waist-to-hip ratio, $\mathrm{HbA}_{1 \mathrm{c}}$, fasting plasma glucose, TC, HDL-cholesterol, LDL-cholesterol, TG, eGFR, white cell count, blood haemoglobin and diabetic

Table 1 Baseline clinical and metabolic characteristics of 2,838 Chinese type 2 diabetic patients with or without chronic HBV infection

\begin{tabular}{|c|c|c|c|c|}
\hline & HbsAg-positive $(n=286)$ & HbsAg-negative $(n=2,552)$ & All patients $(n=2,838)$ & $p$ value* \\
\hline Age (years) & $57.6 \pm 12.0$ & $60.0 \pm 12.9$ & $59.7 \pm 12.9$ & 0.004 \\
\hline Age at onset of diabetes (years) & $51.0 \pm 11.5$ & $53.7 \pm 12.7$ & $53.5 \pm 12.6$ & 0.001 \\
\hline Duration of diabetes (years) & $6.6 \pm 6.0$ & $6.4 \pm 6.0$ & $6.4 \pm 6.0$ & 0.50 \\
\hline Smoking (current and ex) & $95(33 \%)$ & $698(27 \%)$ & $793(28 \%)$ & 0.04 \\
\hline Male sex & $144(50 \%)$ & $1,025(40 \%)$ & $1,169(41 \%)$ & 0.001 \\
\hline Systolic blood pressure (mmHg) & $134 \pm 21$ & $137 \pm 22$ & $136 \pm 22$ & 0.04 \\
\hline Diastolic blood pressure (mmHg) & $79 \pm 11$ & $79 \pm 11$ & $79 \pm 11$ & 0.73 \\
\hline \multicolumn{5}{|l|}{ BMI $\left(\mathrm{kg} / \mathrm{m}^{2}\right)$} \\
\hline Male & $24.0 \pm 3.1$ & $24.8 \pm 3.8$ & $24.7 \pm 3.7$ & 0.002 \\
\hline Female & $25.6 \pm 3.6$ & $25.0 \pm 4.0$ & $25.1 \pm 4.0$ & 0.11 \\
\hline \multicolumn{5}{|l|}{ WHR } \\
\hline Male & $0.90 \pm 0.06$ & $0.91 \pm 0.06$ & $0.91 \pm 0.06$ & 0.01 \\
\hline Female & $0.86 \pm 0.06$ & $0.87 \pm 0.07$ & $0.87 \pm 0.07$ & 0.03 \\
\hline White cell count $\left(\times 10^{9}\right)$ & $7.1 \pm 2.1$ & $7.2 \pm 1.9$ & $7.2 \pm 1.9$ & 0.26 \\
\hline Fasting plasma glucose $(\mathrm{mmol} / \mathrm{l})$ & $9.3 \pm 4.1$ & $8.9 \pm 3.3$ & $8.9 \pm 3.4$ & 0.05 \\
\hline $\mathrm{HbA}_{1 \mathrm{c}}(\%)$ & $7.9 \pm 2.0$ & $7.6 \pm 1.9$ & $7.7 \pm 1.9$ & 0.048 \\
\hline $\mathrm{HbA}_{1 \mathrm{c}}>8 \%$ & $115(40 \%)$ & $871(34 \%)$ & $986(35 \%)$ & 0.04 \\
\hline Total cholesterol (mmol/l) & $5.2 \pm 1.2$ & $5.5 \pm 1.2$ & $5.5 \pm 1.2$ & 0.001 \\
\hline HDL-cholesterol (mmol/l) & $1.3 \pm 0.4$ & $1.3 \pm 0.4$ & $1.3 \pm 0.4$ & 0.82 \\
\hline LDL-cholesterol (mmol/l) & $3.3 \pm 1.1$ & $3.5 \pm 1.0$ & $3.4 \pm 1.0$ & 0.02 \\
\hline Triglyceride (mmol/l) & $1.16(0.82-1.73)$ & $1.36(0.94-2.05)$ & $1.34(0.92-2.01)$ & 0.0002 \\
\hline Spot urine ACR (mg/mmol) & $1.65(0.74-7.50)$ & $1.94(0.80-9.55)$ & $1.88(0.79-9.40)$ & 0.25 \\
\hline Plasma creatinine $(\mu \mathrm{mol} / \mathrm{l})$ & $75(62-90)$ & $72(62-88)$ & $72(60-88)$ & 0.043 \\
\hline eGFR $\left(\mathrm{ml} \cdot \mathrm{min}^{-1} \cdot 1.73 \mathrm{~m}^{-2}\right)$ & $89(74-106)$ & $90(73-108)$ & $90(73-107)$ & 0.94 \\
\hline Diabetic retinopathy & $80(28 \%)$ & $571(22 \%)$ & $651(23 \%)$ & 0.048 \\
\hline Diabetic nephropathy & $63(22 \%)$ & $631(25 \%)$ & $694(24 \%)$ & 0.14 \\
\hline Peripheral sensory neuropathy & $56(20 \%)$ & $511(21 \%)$ & $567(21 \%)$ & 0.93 \\
\hline Macrovascular complications & $34(12 \%)$ & $307(12 \%)$ & $341(12 \%)$ & 0.25 \\
\hline Insulin therapy & $43(15 \%)$ & $254(10 \%)$ & $297(10 \%)$ & 0.008 \\
\hline Lipid-lowering drugs & $56(20 \%)$ & $702(28 \%)$ & $758(27 \%)$ & 0.016 \\
\hline Antihypertensive drugs & $64(22 \%)$ & $684(27 \%)$ & $748(26 \%)$ & 0.27 \\
\hline
\end{tabular}

Mean \pm SD or median (interquartile range) or number (\%) as appropriate

*Adjusted for age, sex, age at onset of diabetes, smoking, blood pressure, BMI, WHR, HbA ${ }_{1 \mathrm{c}}$, fasting plasma glucose, TC, HDLcholesterol, LDL-cholesterol, TG 
complications as covariates. A $p$ value $<0.05$ (two-tailed) was considered to be significant.

\section{Results}

In this consecutive cohort of 2,838 Chinese patients with type 2 diabetes, the mean age was $59.7 \pm 12.9$ years and $1,169(41 \%)$ were male. The mean duration of diabetes was $6.4 \pm 6.0$ years. A total of 286 patients $(10.1 \%)$ had chronic HBV infection which was comparable to the prevalence in the local population [2]. Table 1 summarises the clinical and metabolic profiles of these subjects at baseline. Patients with HBV infection were younger and had earlier onset of disease than those without HBV infection. The mean difference in disease duration between the two groups was 2.7 years (95\% CI 1.2-4.3; $p=0.001)$.

At baseline, patients with concomitant HBV infection tended to have worse glycaemic control and were more likely to be insulin-treated (43 [15\%] vs 254 [10\%]; $p=0.01$ ) but they had lower systolic blood pressure, TC, LDL-cholesterol and TG, and were less centrally obese than those without HBV infection. The difference in lipid profile remained significant after adjusting for the use of lipid-lowering agents. Fewer HBV-infected patients took statins or fibrates than un-infected patients (19.6 vs 27.5\%; $p=0.01)$. At baseline, more HBV-infected patients had retinopathy but the rates of other complications, including albuminuria, peripheral sensory neuropathy and macrovascular complications, were similar between the two groups (Table 1). Only six patients in the entire cohort had renal biopsies, with three patients being confirmed as having diabetic glomerulosclerosis, one as having diabetic glomerulosclerosis with crescentic glomerulonephritis, one as having hypertensive nephrosclerosis and one as having IgA nephropathy.

Among the $286 \mathrm{HBV}$-infected patients, 58 had their blood checked for HBeAg and of these, ten were positive for HBeAg. Nearly 50\% $(n=142)$ underwent an ultrasound scan of the abdomen with 41 patients having radiological features of liver cirrhosis. Among $286 \mathrm{HBV}$-infected patients, 241 had their blood checked for platelet count at baseline, 12 had a platelet count $<100 \times 10^{9} / 1$. Of the 127 patients who had both platelet count and ultrasound scan findings, eight had radiological evidence of cirrhosis and a platelet count $>100 \times 10^{9} / 1$, indicating clinically significant cirrhosis.

After a median follow-up of 3.5 years (interquartile range 1.7-5.9 years), 25 (8.7\%) HBV-infected and 163 (6.4\%) non-HBV-infected type 2 diabetes patients devel-

Table 2 Factors associated with increased risk of renal endpoint defined as need for dialysis, doubling of serum creatinine or serum creatinine $\geq 500 \mu \mathrm{mol} / 1$

\begin{tabular}{|c|c|c|c|}
\hline Factors & Hazard ratio & $95 \%$ confidence interval & $p$ value \\
\hline \multicolumn{4}{|l|}{ Univariate analysis } \\
\hline HBsAg-positive status & 2.79 & $1.03-7.56$ & 0.04 \\
\hline Male sex & 1.00 & $0.43-2.34$ & 1.00 \\
\hline Age & 1.01 & $0.97-1.04$ & 0.73 \\
\hline Age of onset of diabetes mellitus & 1.05 & $0.99-1.11$ & 0.11 \\
\hline Smoking (current and ex) & 0.60 & $0.26-1.40$ & 0.23 \\
\hline Systolic blood pressure & 1.03 & $1.01-1.04$ & 0.002 \\
\hline Diastolic blood pressure & 1.05 & $1.02-1.09$ & 0.004 \\
\hline BMI & 0.95 & $0.85-1.07$ & 0.40 \\
\hline WHR & 3.50 & $0.03-400.40$ & 0.61 \\
\hline $\mathrm{HbA}_{1 \mathrm{c}}$ & 1.13 & $0.95-1.34$ & 0.18 \\
\hline Fasting plasma glucose & 1.05 & $0.94-1.17$ & 0.38 \\
\hline Total cholesterol & 1.72 & $1.37-2.17$ & $<0.0001$ \\
\hline HDL-cholesterol & 0.70 & $0.21-2.41$ & 0.57 \\
\hline LDL-cholesterol & 2.08 & $1.56-2.77$ & $<0.0001$ \\
\hline Triglyceride & 1.60 & $0.87-2.94$ & 0.13 \\
\hline White cell count & 1.00 & $0.81-1.23$ & 1.00 \\
\hline eGFR * & 0.016 & $0.004-0.058$ & $<0.0001$ \\
\hline Urine ACR & 2.59 & $2.07-3.25$ & $<0.0001$ \\
\hline \multicolumn{4}{|l|}{ Multivariate analysis } \\
\hline HbsAg-positive status & 4.53 & $1.11-18.58$ & 0.036 \\
\hline Diastolic blood pressure & 1.089 & $1.007-1.177$ & 0.032 \\
\hline eGFR* & 0.076 & $0.008-0.721$ & 0.025 \\
\hline Urine ACR & 1.79 & $1.17-2.74$ & 0.007 \\
\hline
\end{tabular}

Based on the Modification of Diet in Renal Disease equation [20]

Multivariate analysis was performed by the Cox regression model with all the factors listed 
oped end-stage renal disease (ESRD). Patients who developed ESRD were more likely to have chronic HBV infection (16.1 vs $10.1 \%$ ), retinopathy (74.2 vs $20.9 \%$ ) and albuminuria (96.3 vs $30.3 \%$ ) at baseline. On univariate analysis, HBsAg, systolic and diastolic blood pressure, retinopathy, TC, LDL-cholesterol, eGFR and ACR were predictors of ESRD. After controlling for these risk factors, only eGFR, diastolic blood pressure and chronic HBV infection were independent predictors of ESRD on multivariate analysis (Table 2). Using Kaplan-Meier analysis, patients with chronic HBV infection had higher cumulative frequencies of $\operatorname{ESRD}(p=0.001$, Fig. 1a) but lower cardiovascular endpoints $(p=0.53$, Fig. $1 \mathrm{~b})$ than non$\mathrm{HBV}$-infected patients. Among the 41 patients with liver cirrhosis on imaging and eight patients with clinical cirrhosis (imaging and platelet count below $100 \times 10^{9} / 1$, only one patient had ESRD. In the HBV-positive group, only four had acute renal failure and liver decompensation, suggesting possible hepatorenal syndrome. These were all terminally ill patients with hepatocellular carcinoma and sepsis.

During the observational period, 130 patients died. The death rate was higher in the HBV-positive group than the HBV-negative group $(7.3 \%$ vs $4.3 \% ; p<0.0001)$ with a hazard ratio of $2(1.2-3.4, p=0.007$; Fig. $1 \mathrm{c})$. The excess mortality was mainly attributed to liver-related deaths and hepatocellular carcinoma. Deaths from cardiovascular events and malignancy other than hepatocellular carcinoma were similar between both groups (Table 3 ).

\section{Discussion}

In this cohort of Chinese patients with type 2 diabetes, chronic HBV infection was associated with an almost three-fold increased risk of ESRD. After adjusting for potential confounding factors, this risk ratio increased further to 4.5 along with other predictors including eGFR, albuminuria and diastolic blood pressure. Despite the high prevalence of both HBV infection and type 2 diabetes on a global basis, there is a paucity of clinical studies addressing the possible risk association between these two major public health problems. To our knowledge, this is the first study showing the increased risk of ESRD in type 2 diabetics with chronic HBV infection.

In patients with chronic $\mathrm{HCV}$ infection, the increased prevalence of type 2 diabetes was hypothesised to be mediated through insulin resistance, partly because of an increased release of inflammatory mediators such as TNF- $\alpha$ [12]. In this study, our data did not demonstrate increased frequency of chronic HBV infection in patients with type 2 diabetes. Nevertheless, there is indirect evidence suggesting that chronic HBV infection may increase the risk of chronic inflammation and insulin resistance, which then interact with other factors to influence the clinical course of type 2 diabetes. In a study involving 767 pregnant Chinese women in Hong Kong, HBsAg carrier status was associated with a 3.5-fold increased risk of gestational diabetes [15]. The HbsAgpositive women with gestational diabetes had higher serum ferritin levels than the HbsAg-negative women. The increased ferritin level might be the result of chronic inflammation or increased hepatocellular release secondary to tissue necrosis. In a selected cohort of type 2 diabetic patients with overt nephropathy, we observed a five-fold increased risk of cardiovascular events among patients with chronic HBV infection over a median follow-up period of 2 years [16].

In a recent meta-analysis, Asians were found to have a lower risk of developing cardiovascular disease but a higher risk of renal disease than Caucasians $[22,23]$. In the present analysis, patients with chronic HBV infection were younger, had earlier onset of disease and were more likely to be insulin-treated and have retinopathy at baseline. These findings lend support to the notion that chronic HBV infection might adversely affect the metabolic milieu of subjects who are at risk of developing type 2 diabetes. Given the importance of glycaemia as one of the key determinants of progression of albuminuria and development of ESRD [24, 25], it is plausible that chronic HBV infection might worsen insulin resistance and hyperglycaemia, which interact with chronic inflammation to increase the risk of ESRD in our patients. In support of our hypothesis, HBsAg was found to be an independent risk factor for ESRD along with albuminuria, eGFR, diastolic blood pressure and anaemia. Although we have reported the association between white cell count and diabetic complications in a cross-sectional survey [26], white cell count was not found to be a predictor in this prospective analysis. More sensitive inflammatory markers such as high-sensitivity $\mathrm{C}$-reactive protein may need to be measured to clarify the role of chronic inflammation in these observations.

Table 3 Causes of death in type 2 diabetic patients with chronic HBV infection compared to non-HBV-infected diabetes patients after a mean follow-up period of 3.5 years (range 1.7-5.9 years)

\begin{tabular}{|c|c|c|c|c|}
\hline Causes of death & HBsAg-positive $(n=286)$ & HbsAg-negative $(n=2,552)$ & Overall $(n=2,838)$ & $p$ value \\
\hline Cardiovascular death & $2(0.7 \%)$ & $28(1.1 \%)$ & 30 & 0.76 \\
\hline Renal death & $2(0.7 \%)$ & $8(0.3 \%)$ & 10 & 0.27 \\
\hline Liver failure or hepatocellular carcinoma & $11(3.8 \%)$ & $7(0.3 \%)$ & 18 & $<0.0001$ \\
\hline Malignancy other than hepatocellular carcinoma & $2(0.7 \%)$ & $26(1 \%)$ & 28 & 1 \\
\hline Others & $4(1.4 \%)$ & $40(1.6 \%)$ & 44 & 1 \\
\hline Total & 21 & 109 & 130 & 0.018 \\
\hline
\end{tabular}




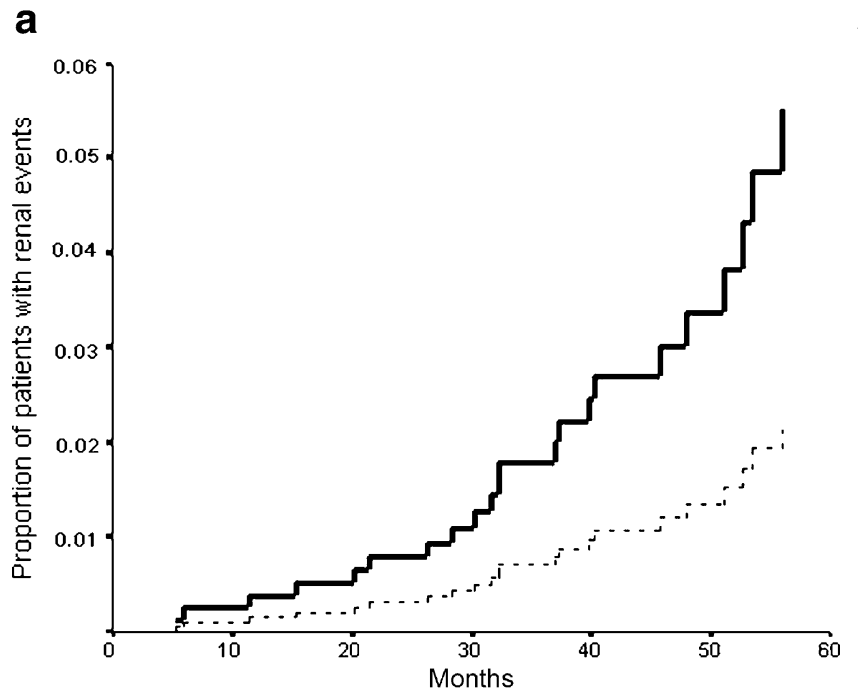

b

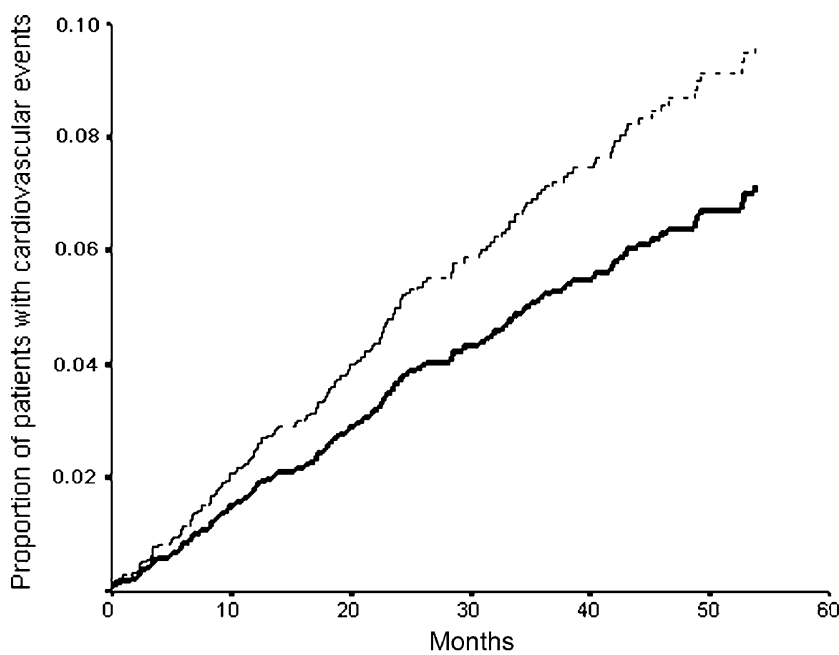

C

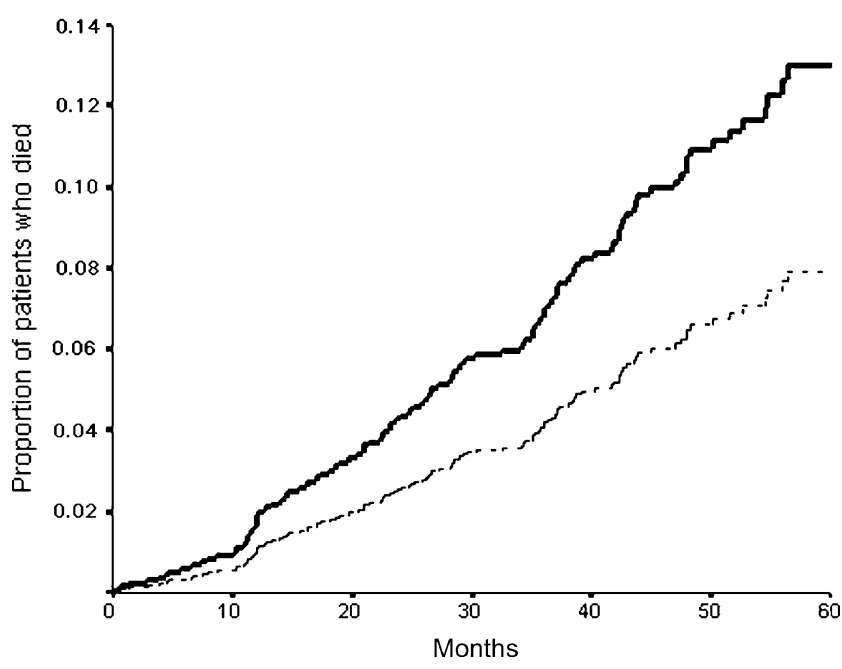

In a separate and selected cohort of type 2 diabetes patients with overt nephropathy, we have reported the risk association between chronic HBV infection and cardiovascular events [16]. Of note, the cohort studied [16] was a
Fig. 1 Kaplan-Meier curves showing the cumulative frequencies of renal, cardiovascular endpoints and all-cause mortality in patients with and without chronic hepatitis B infection. Mean follow-up duration was 3.5 years (range 1.7-5.9 years). A Cox-proportional hazard model was used with adjustment for age, sex, duration of diabetes, blood pressure, BMI, WHR, fasting plasma glucose, $\mathrm{HbA}_{1 \mathrm{c}}$, TC, HDL-cholesterol, LDL-cholesterol, TG and estimated glomerular filtration rate at baseline. Solid line, HBV-positive group; dotted line, HBV-negative group. a Proportion of patients with renal events, defined as serum creatinine $\geq 500 \mu \mathrm{mol} / \mathrm{l}$, doubling of serum creatinine or requiring renal dialysis: $25(8.7 \%)$ in the HBV-positive and $163(6.4 \%)$ in the HBV-negative group; $p=0.001$. b Proportion of patients with cardiovascular events: $13(4.5 \%)$ in the HBVpositive group and $157(6.2 \%)$ in the HBV-negative group; $p=0.53$. c Overall death rate, $21(7.3 \%)$ in the HBV-positive group and 109 $(4.3 \%)$ in the HBV-negative group; $p<0.0001$

different population with higher cardiovascular risk because of renal impairment and heavy proteinuria as inclusion criteria. In this current large cohort of type 2 diabetics with relatively low cardiovascular risk profiles, HBV-positive patients had lower incidence of cardiovascular endpoints than HBV-negative patients, albeit the difference was statistically insignificant. This may be partly because of the lower degree of obesity and less adverse lipid and blood pressure control in patients with chronic HBV infection than the non-infected patients.

In this cohort, approximately $6 \%$ of the HBV-infected patients had clinical evidence of liver cirrhosis, as defined by the presence of radiological features and thrombocytopenia. In an unselected clinic-based population with chronic liver disease in Taiwan, the annual incidence of liver cirrhosis was reported to be $2.4 \%$ in HBV-positive patients and $1.3 \%$ in HBV-negative subjects [27]. The annual incidence of hepatocellular carcinoma among cirrhotic patients was reported to be $2-3 \%$ [28]. There is now evidence suggesting that diabetes is an independent risk factor for chronic liver disease and hepatocellular carcinoma [29]. This may be explained by the possible accelerating effects of chronic hyperglycaemia on the rate of hepatic fibrosis by inducing hepatic steatosis in patients with chronic viral hepatitis, thus further increasing their risk of cirrhosis [30]. Taken together, these findings may explain the relatively high rate of death from cirrhosis and hepatocellular carcinoma in our type 2 diabetic cohort.

This study has several limitations. First, the effect of viral load was not assessed in our patients. Only a small percentage of $\mathrm{HBV}$-infected patients had their $\mathrm{HBeAg}$ status tested and HBV DNA levels were not checked. Hence, the associations between the severity of chronic HBV infection and diabetic complications could not be fully addressed. Second, HBV infection was known to be associated with glomerulonephritis, which might be a confounding factor for the increased risk of ESRD in patients with chronic HBV infection [31]. However, renal biopsy was performed in only a small number of our patients so this question could not be addressed. Nevertheless, given the higher rate of retinopathy in patients with HBV infection and/or ESRD, the contribution of diabetic microvasculopathy cannot be excluded. Third, only half of the HBV-infected patients underwent ultrasound scan and liver cirrhosis might be a confounding factor for increased 
risk of ESRD caused by hepatorenal syndrome. However, since abnormal liver function tests or clinical features of cirrhosis were the main indications for an ultrasound scan of the liver in our subjects, those who did not undergo ultrasound imaging were not likely to have significant derangement of liver function or cirrhosis. Besides, among patients who underwent ultrasound imaging, only one of all the cirrhotic patients had ESRD, suggesting that the confounding effect of cirrhosis was low if any. Fourth, the possibility of potential concurrent $\mathrm{HCV}$ infection could not be excluded. Lastly, our study design could not determine the mechanism of the 'premature' ESRD in HBV-positive diabetic patients and further studies are required to elucidate the underlying pathogenetic mechanisms.

In conclusion, our data provide additional insights into the associations between chronic HBV infection and type 2 diabetes and suggest that chronic HBV infection predicts the occurrence of ESRD, independent of other conventional risk factors, in Chinese patients with type 2 diabetes. Further studies are required to confirm the reproducibility of our results and to explore the underlying pathogenetic mechanisms. Early detection of HBV status and close surveillance of complications, especially renal function, are important in managing diabetic patients who live in areas where HBV is endemic or who are at risk of developing chronic HBV infection.

Acknowledgements We thank all the medical and nursing staff at the Diabetes Mellitus and Endocrine Centre, Prince of Wales Hospital for their commitment and dedication in implementing the structured diabetes care protocol and its continuous quality improvement. This study was partially supported by funding from Li Ka Shing Institute of Health Sciences, The Chinese University of Hong Kong.

\section{References}

1. Janus ED, Watt NM, Lam KS et al (2000) The prevalence of diabetes, association with cardiovascular risk factors and implications of diagnostic criteria (ADA 1997 and WHO 1998 ) in a 1996 community-based population study in Hong Kong Chinese. Hong Kong Cardiovascular Risk Factor Steering Committee. American Diabetes Association. Diabet Med 17:741-745

2. No authors listed. (2003) Surveillance of viral hepatitis in Hong Kong - 2003 Update Report. Department of Health, Hong Kong SAR. http://www.chp.gov.hk/files/pdf/hep 2005020301. pdf (accessed 25 August, 2005)

3. Kruszynska YT, McIntyre N (1991) Carbohydrate metabolism. In: McIntyre N, Benhamou JP, Bircher J, Rizzetto M, Rodes J (eds) Oxford textbook of clinical hepatology. Oxford University Press, Oxford, pp 129-143

4. Allison ME, Wreghitt T, Palmer CR, Alexander GJ (1994) Evidence for a link between hepatitis $\mathrm{C}$ virus infection and diabetes mellitus in a cirrhotic population. J Hepatol 21:1135-1139

5. Caronia S, Taylor K, Pagliaro L et al (1999) Further evidence for an association between non-insulin-dependent diabetes mellitus and chronic hepatitis $\mathrm{C}$ virus infection. Hepatology 30:1059-1063
6. Grimbert S, Valensi P, Levy-Marchal C et al (1996) High prevalence of diabetes mellitus in patients with chronic hepatitis C. A case-control study. Gastroenterol Clin Biol 20:544-548

7. Knobler H, Schihmanter R, Zifroni A, Fenakel G, Schattner A (2000) Increased risk of type 2 diabetes in noncirrhotic patients with chronic hepatitis $\mathrm{C}$ virus infection. Mayo Clin Proc 75:355-359

8. Mason AL, Lau JY, Hoang N et al (1999) Association of diabetes mellitus and chronic hepatitis $\mathrm{C}$ virus infection. Hepatology 29:328-333

9. Mehta SH, Brancati FL, Sulkowski MS, Strathdee SA, Szklo M, Thomas DL (2000) Prevalence of type 2 diabetes mellitus among persons with hepatitis $\mathrm{C}$ virus infection in the United States. Ann Intern Med 133:592-599

10. Shintani Y, Fujie H, Miyoshi H et al (2004) Hepatitis C virus infection and diabetes: direct involvement of the virus in the development of insulin resistance. Gastroenterology 126:840-848

11. Antonelli A, Ferri C, Fallahi P et al (2005) Hepatitis C virus infection: evidence for an association with type 2 diabetes. Diabetes Care 28:2548-2550

12. Knobler H, Schattner A (2005) TNF-a, chronic hepatitis C and diabetes: a novel triad. QJM 98:1-6

13. Crook ED, Penumalee S, Gavini B, Filippova K (2005) Hepatitis $\mathrm{C}$ is a predictor of poorer renal survival in diabetic patients. Diabetes Care 28:2187-2191

14. Soma J, Saito T, Taguma Y et al (2000) High prevalence and adverse effect of hepatitis $\mathrm{C}$ virus infection in type II diabeticrelated nephropathy. J Am Soc Nephrol 11:690-699

15. Lao TT, Tse KY, Chan LY, Tam KF, Ho LF (2003) HBsAg carrier status and the association between gestational diabetes with increased serum ferritin concentration in Chinese women. Diabetes Care 26:3011-3016

16. Lo MK, Lee KF, Chan NN et al (2004) Effects of gender, Helicobacter pylori and hepatitis B virus serology status on cardiovascular and renal complications in Chinese type 2 diabetic patients with overt nephropathy. Diabetes Obes Metab $6: 223-230$

17. Piwernetz K, Home PD, Snorgaard O, Antsiferov M, Staehr-Johansen K, Krans M (1993) Monitoring the targets of the St Vincent Declaration and the implementation of quality management in diabetes care: the DIABCARE initiative. The DIABCARE Monitoring Group of the St Vincent Declaration Steering Committee. Diabet Med 10:371-377

18. Laakso M, Pyorala K (1985) Age of onset and type of diabetes. Diabetes Care 8:114-117

19. Mogensen CE, Vestbo E, Poulsen PL et al (1995) Microalbuminuria and potential confounders. A review and some observations on variability of urinary albumin excretion. Diabetes Care 18:572-581

20. Levey AS, Bosch JP, Lewis JB, Greene T, Rogers N, Roth D (1999) A more accurate method to estimate glomerular filtration rate from serum creatinine: a new prediction equation. Modification of Diet in Renal Disease Study Group. Ann Intern Med 130:461-470

21. Friedewald WT, Levy RI, Fredrickson DS (1972) Estimation of the concentration of low-density lipoprotein cholesterol in plasma, without use of the preparative ultracentrifuge. Clin Chem 18:499-502

22. Karter AJ, Ferrara A, Liu JY, Moffet HH, Ackerson LM, Selby JV (2002) Ethnic disparities in diabetic complications in an insured population. JAMA 287:2519-2527

23. Wu AY, Kong NC, de Leon FA et al (2005) An alarmingly high prevalence of diabetic nephropathy in Asian type 2 diabetic patients: the MicroAlbuminuria Prevalence (MAP) Study. Diabetologia 48:17-26

24. No authors listed (1998) Intensive blood-glucose control with sulphonylureas or insulin compared with conventional treatment and risk of complications in patients with type 2 diabetes (UKPDS 33). UK Prospective Diabetes Study (UKPDS) Group. Lancet 352:837-853 
25. Fioretto P, Steffes MW, Sutherland DE, Goetz FC, Mauer M (1998) Reversal of lesions of diabetic nephropathy after pancreas transplantation. N Engl J Med 339:69-75

26. Tong P, Lee K, So W et al (2004) Association of white blood cell counts with macrovascular and microvascular complications in Chinese patients with type 2 diabetes. Diabetes Care 27:216-222

27. Liaw YF, Tai DI, Chu CM, Chen TJ (1988) The development of cirrhosis in patients with chronic type B hepatitis: a prospective study. Hepatology 8:493-496
28. Liaw YF, Lin DY, Chen TJ, Chu CM (1989) Natural course after the development of cirrhosis in patients with chronic type B hepatitis: a prospective study. Liver 9:235-241

29. El-Serag HB, Tran T, Everhart JE (2004) Diabetes increases the risk of chronic liver disease and hepatocellular carcinoma. Gastroenterology 126:460-468

30. Gordon A, McLean CA, Pedersen JS, Bailey MJ, Roberts SK (2005) Hepatic steatosis in chronic hepatitis B and C: predictors, distribution and effect on fibrosis. J Hepatol 43:38-44

31. Lai KN, Li PK, Lui SF et al (1991) Membranous nephropathy related to hepatitis B virus in adults. N Engl J Med 324:1457-1463 